\title{
Post Surgical Spinal Evaluation
}

One of the most challenging areas of diagnosis is to be found in acquiring and interpreting medical images in a patient who has undergone surgery for spinal degenerative disease. This may have involved partial or complete laminectomy, discectomy (surgical removal of the native and/or herniated portions of the intervertebral disc), intervertebral bony fusion and/or spinal instrumentation. The imaging findings may be either of an expected or unexpected nature. In some instances, the treated tissues may be left with benign scarring; in other cases, there may be a recurrence of disease or the appearance of a different type of disease process engendered by the surgical procedure. In still other situations, the observation may represent a true acute/subacute complication of the operation. All of these possibilities complicate medical image analysis (Ghazi et al., 1992; Jinkins, 1993; Jinkins et al., 1993; Grane et al., 1998; Shafaiae et al., 1997; Van Goethem et al., 1997).

In order to critically evaluate the posttherapeutic patient, it is imperative to understand several factors in reasonably specific detail. These factors include (a) the primary clinical syndrome and diagnosis, (b) the surgical treatment(s) undergone by the patient, (c) the elapsed time since the operative procedure(s), and (d) the current clinical syndrome. The answers to these questions will in large part determine which imaging modality or modalities are chosen for the patient evaluation, how the images are acquired, and whether or not an enhancing agent is used.

\section{CONVENTIONAL AND FAST SPIN ECHO ACQUISITIONS}

Progressive improvements in magnetic resonance (MR) imaging have substantially improved the ability of the medical imaging physician to critically analyze the postoperative lumbosacral spine following surgery for degenerative spinal disease. Compared to other imaging methods, MR images acquired in multiple planes have superior diagnostic potential, in part because of their generally greater spatial and contrast resolution characteristics for many tissues. Nevertheless, the similarity of some postsurgical pathologic processes in regard to MR signal intensity often makes even this imaging technique somewhat difficult to interpret. The development of intravenously (i.v.) administered paramagnetic MR contrast agents (e.g., gadolinium) has materially assisted the diagnostic sensitivity and specificity of MR imaging in the evaluation of the failed back surgery syndrome (FBSS) because of the improvement in differential contrast enhancement afforded by these agents.

In general, $T_{2}$-weighted fast spin-echo images are superior to conventional spin-echo images of the lumbosacral spine in part because of improved image quality resulting from superior spatial resolution and reduced motion artifact. Sagittal (with fat suppression) and transverse (without fat suppression) fast spin-echo $T_{2}$-weighted images are helpful in assessing neural foramen narrowing, central spinal canal and lateral recess spinal stenosis, hydration status of the intervertebral disc, abnormal signal intensity of the disc and cancellous bone and signal intensity of abnormal intra- or perispinal soft tissue masses (e.g., disc herniation, epidural scar, epidural phlegmon, epidural abscess, posterior facet joint synovial cyst). Sagittal and transverse $T_{1}$-weighted spin-echo images obtained before and immediately after the bolus i.v. injection of a gadolinium product are almost imperative in the evaluation of the postoperative lumbar spine, in order to evaluate the integrity of the blood-nerve barrier in certain cases of suspected compressive radiculopathy, or in cases of epidural masses of ambiguous etiology (e.g., epidural infection versus intervertebral disc fragment versus epidural fibrosis). 
Table A8.8.1 Equipment Parameters for Spine Imaging in Cases of Postsurgical Evaluation

\begin{tabular}{|c|c|}
\hline Coil type & $\begin{array}{l}\text { Cervical, thoracic, lumbar: phased array, } \\
\text { surface coil (or other depending upon } \\
\text { machine compatibility and availability) }\end{array}$ \\
\hline Peripheral gating & Thoracic spine only (optional) \\
\hline Respiratory gating & Thoracic spine only (optional) \\
\hline Flow compensation pulse & Any level (if available) \\
\hline Use of contrast agents & Yes $^{a}$ \\
\hline
\end{tabular}

${ }^{a}$ In emergency situations, contrast agents may not be necessary.

Fat-suppression techniques can also often be used after i.v. gadolinium administration because they improve the relative intensity and homogeneity of contrast enhancement when it exists. However, it may make the critical interpretation of possible abnormal postoperative intrathecal nerve root enhancement (i.e., sterile radiculitis) difficult or impossible because small degrees of apparently normal nerve root enhancement are not infrequently observed when fat suppression MR imaging techniques are used with i.v. gadolinium enhancement.

The following sequences comprise the preferred protocol for high-field MR machines. On some machines alternate gradient recalled echo acquisitions may be more desirable. This entire protocol should take 45 to 50 min to complete.

Table A8.8.1 lists the hardware necessary to perform the procedure, along with appropriate parameters. The available gradient strength will depend on the scanner, and the echo times given in other tables will be varied accordingly (the smaller the gradient strength, the longer the echo time for a particular scan).

NOTE: Be sure that technicians and nurses have immediate access to any emergency equipment that may be relevant to a given study, or that may be needed for a particular patient, such as crash carts or oxygen.

\section{Materials}

Normal saline $(0.9 \% \mathrm{NaCl})$ sterile

Extravascular contrast agent (e.g., Mangevist, Omniscan, or Prohance)

\section{Set up patient and equipment}

1. Interview (screen) the patient to ensure that he or she has no contraindications such as cardiac pacemakers or other implants containing ferromagnetic materials. Also be sure to find out if the patient has any health conditions that may require the presence of special emergency equipment during the scanning procedure, or necessitate any other precautions.

Generally standard screening forms are used for all patients scanned in a magnetic resonance system.

The presence of any ferromagnetic metals may be a health hazard to the patient when he or she is inside the magnet, and will also affect the imaging. If in doubt as to the exact composition of the items, it is best to exclude patients with any metal implants; see Shellock (1996) for discussion of what implants may be safely scanned using magnetic resonance.

Patients may be accompanied into the magnet room by a friend or family member, who can sit in the room during the scan and comfort the patient as needed. This companion must be screened as well to ensure the absence of loose metal objects on the body or clothing. 
Table A8.8.2 Primary Clinical Imaging Parameters for Sequence 1 (Pilot Scan)

\begin{tabular}{ll}
\hline Patient position & Supine \\
Scan type & Gradient echo \\
Imaging plane (orientation) & Transverse \\
Central slice or volume center & Centered on: \\
& Cervical spine: thyroid cartilage \\
& Thoracic spine: nipple line \\
Echo time $\left(T_{\mathrm{E}}\right)$ & Lumbar spine: iliac crest \\
Repeat time $\left(T_{\mathrm{R}}\right)$ & As short as possible \\
Flip angle $(\mathrm{FA})$ & As short as possible \\
Fields of view $(\mathrm{FOV}, \mathrm{FOV}$ & \\
& $15^{\circ}$ \\
& Cervical: $240 \mathrm{~mm}, 240 \mathrm{~mm}$ \\
Resolution $(\Delta x, \Delta y)$ & Thoracic: $320 \mathrm{~mm}, 320 \mathrm{~mm}$ \\
& Lumbosacral: $280 \mathrm{~mm}, 280 \mathrm{~mm}$ \\
Number of data points collected $\left(N_{\mathrm{x}}, N_{\mathrm{y}}\right)$ & Cervical: $0.94 \mathrm{~mm}, 0.94 \mathrm{~mm}$ \\
Display matrix $\left(D_{\mathrm{x}}, D_{\mathrm{y}}\right)$ & Thoracic: $1.25 \mathrm{~mm}, 1.25 \mathrm{~mm}$ \\
Slice thickness $(\Delta z)$ & 256,256 \\
Number of slices & 5 mm \\
Slice gap & Variable \\
Number of acquisitions $\left(N_{\mathrm{acq}}\right)$ & Not applicable \\
Scan time & 1 \\
\hline
\end{tabular}

2. If the procedure is a research protocol, have the patient sign any necessary consent form.

3. Have the patient remove all jewelry and change into a gown to eliminate any metal that might be found in clothing.

4. Inform the patient about what will occur during the procedure, what he or she will experience while in the magnet, and how to behave, including the following:

a. If earphones or headphones are used to protect the ears from the loud sounds produced by the gradients, the patient will be asked to wear these, but will be able to communicate with you at any time during the imaging.

b. The patient will be given a safety squeeze-bulb or similar equipment to request assistance at any time (demonstrate how this works).

c. For good results the patient should not talk, and should avoid or minimize swallowing or other movement during each scan-i.e., as long as the banging sounds continue. Between scans, talking and swallowing are allowed in most cases, but should be avoided when comparative positional studies are being performed; the patient will be informed when this is the case.

d. Nevertheless, the patient may call out at any time if he or she feels it necessary.

5. Have the patient mount onto the table. Either before or right after the patient lies down, set up any triggering devices or other monitoring equipment that is to be used.

6. Center the coil over the region where the key information is desired.

Make sure that the body is constrained to prevent motion, especially if high-resolution scans are to be run. 
Table A8.8.3 Primary Clinical Imaging Parameters for Sequence 2 ( $T_{1}$-Weighted Image)

\begin{tabular}{|c|c|}
\hline Patient position & Supine \\
\hline Scan type & Conventional spin echo \\
\hline Imaging plane (orientation) & Sagittal \\
\hline \multirow[t]{2}{*}{ Central slice or volume center } & Centered on: \\
\hline & $\begin{array}{l}\text { Cervical: 3rd cervical vertebra } \\
\text { Thoracic: 6th thoracic vertebra } \\
\text { Lumbar: 3rd lumbar vertebra }\end{array}$ \\
\hline Echo time $\left(T_{\mathrm{E}}\right)$ & $10 \mathrm{msec}$ \\
\hline Repeat time $\left(T_{\mathrm{R}}\right)$ & $500 \mathrm{msec}$ \\
\hline Flip angle (FA) & $90^{\circ}$ \\
\hline Fields of view $\left(\mathrm{FOV}_{\mathrm{x}}, \mathrm{FOV}_{\mathrm{y}}\right)$ & $\begin{array}{l}\text { Cervical: } 240 \mathrm{~mm}, 240 \mathrm{~mm} \\
\text { Thoracic: } 320 \mathrm{~mm}, 320 \mathrm{~mm} \\
\text { Lumbosacral: } 280 \mathrm{~mm}, 280 \mathrm{~mm} \text { (may } \\
\text { use rectangular field of view [e.g., half } \\
\text { or three-quarter field] if available, or } \\
\text { tailor to region of interest) }\end{array}$ \\
\hline Resolution $(\Delta x, \Delta y)$ & $\begin{array}{l}\text { Cervical: } 0.94 \mathrm{~mm}, 0.94 \mathrm{~mm} \\
\text { Thoracic: } 1.25 \mathrm{~mm}, 1.25 \mathrm{~mm} \\
\text { Lumbosacral: } 1.09 \mathrm{~mm}, 1.09 \mathrm{~mm}\end{array}$ \\
\hline Number of data points collected $\left(N_{\mathrm{x}}, N_{\mathrm{y}}\right)$ & 256,256 \\
\hline Display matrix $\left(D_{\mathrm{x}}, D_{\mathrm{y}}\right)$ & 256,256 \\
\hline Slice thickness $(\Delta z)$ & $\begin{array}{l}\text { Cervical: } 3 \mathrm{~mm} \\
\text { Thoracic: } 3 \mathrm{~mm} \\
\text { Lumbar: } 4 \mathrm{~mm}\end{array}$ \\
\hline Number of slices & $\begin{array}{l}\text { As many as needed to cover the region } \\
\text { of interest }\end{array}$ \\
\hline Slice gap & $\begin{array}{l}\text { Cervical: } 0.5 \mathrm{~mm} \\
\text { Thoracic: } 1 \mathrm{~mm} \\
\text { Lumbar: } 1 \mathrm{~mm}\end{array}$ \\
\hline Number of acquisitions $\left(N_{\text {acq }}\right)$ & 2 \\
\hline Flow compensation & Yes (if available) \\
\hline Saturation pulses & $\begin{array}{l}\text { Yes; anterior cervical/thoracic/lumbar } \\
\text { slab to saturate larynx/vessels }\end{array}$ \\
\hline Slice series & $\begin{array}{l}\text { Left to right or the reverse depending } \\
\text { on preference }\end{array}$ \\
\hline Scan time & $\sim 4 \min$ \\
\hline
\end{tabular}

7. If needed, place a pillow or other support under the knees to make the patient more comfortable.

8. Use the centering light to position the patient (cervical spine: thyroid cartilage; thoracic spine: nipple line; spine: iliac crests) and put him or her into the center of the magnet.

Once this step has been performed, so long as the patient does not move on the table, the table itself can be moved and then replaced in the same position as before without jeopardizing the positioning of one scan relative to another.

9. If the patient is unable to hold still, provide an appropriate sedative.

Post Surgical 
Table A8.8.4 Primary Clinical Imaging Parameters for Sequence 3 ( $T_{2}$-Weighted Image, FSE) ${ }^{a}$

\begin{tabular}{|c|c|}
\hline Patient position & Supine \\
\hline Scan type & Fast spin echo \\
\hline Imaging plane (orientation) & Sagittal \\
\hline Central slice or volume center & $\begin{array}{l}\text { Centered on area of interest (as in } \\
\text { sequence } 2 \text {, Table A8.8.3) }\end{array}$ \\
\hline Echo time $\left(T_{\mathrm{E}}\right)$ & $100 \mathrm{msec}$ \\
\hline Echo train length (ETL) & 8 \\
\hline Repeat time $\left(T_{\mathrm{R}}\right)$ & $4000 \mathrm{msec}$ \\
\hline Flip angle (FA) & $90^{\circ}$ \\
\hline Fields of view $\left(\mathrm{FOV}_{\mathrm{x}}, \mathrm{FOV}_{\mathrm{y}}\right)$ & As in sequence 2 , Table A8.8.3 \\
\hline Resolution $(\Delta x, \Delta y)$ & $\begin{array}{l}\text { Cervical: } 0.47 \mathrm{~mm}, 0.47 \mathrm{~mm} \\
\text { Thoracic: } 0.63 \mathrm{~mm}, 0.63 \mathrm{~mm} \\
\text { Lumbosacral: } 0.55 \mathrm{~mm}, 0.55 \mathrm{~mm}\end{array}$ \\
\hline Number of data points collected $\left(N_{\mathrm{x}}, N_{\mathrm{y}}\right)$ & 512,512 \\
\hline Display matrix $\left(D_{\mathrm{x}}, D_{\mathrm{y}}\right)$ & 512,512 \\
\hline Slice thickness $(\Delta z)$ & $\begin{array}{l}\text { Cervical: } 3 \mathrm{~mm} \\
\text { Thoracic: } 3 \mathrm{~mm} \\
\text { Lumbar: } 4 \mathrm{~mm}\end{array}$ \\
\hline Number of slices & Varies with spinal level \\
\hline Slice gap & $\begin{array}{l}\text { Cervical: } 0.5 \mathrm{~mm} \\
\text { Thoracic: } 1 \mathrm{~mm} \\
\text { Lumbar: } 1 \mathrm{~mm}\end{array}$ \\
\hline Number of acquisitions $\left(N_{\text {acq }}\right)$ & 1 \\
\hline Flow compensation & Yes (if available) \\
\hline Saturation pulses & $\begin{array}{l}\text { Yes; anterior cervical/thoracic/lumbar } \\
\text { slabs to saturate larynx/vessels/heart }\end{array}$ \\
\hline Fat suppression & Yes \\
\hline Slice series & $\begin{array}{l}\text { Left to right or the reverse depending } \\
\text { on preference }\end{array}$ \\
\hline Scan time & $\sim 4 \min$ \\
\hline
\end{tabular}

${ }^{\mathrm{a} F S E}$ : fast spin echo.

\section{Sequence 1: Rapid positioning pilot}

10. To validate the patient's position, run the system's pilot (or scout) scan (sequence 1) to ensure correct location of the neck in three dimensions, using the imaging sequence given in Table A8.8.2 or similar parameters.

This sequence usually consists of three orthogonal planes to allow subsequent localization. The images are often also used later to determine where to place the saturation pulses and to set up total coverage of the volume of interest.

NOTE: In an emergency situation (e.g., postoperative acute hemorrhage), only sequences 2 to 5 may be indicated.

\section{Sequence 2: Sagittal $T_{1}$-weighted conventional spin echo}

11. Set the imaging parameters as shown in Table A8.8.3.

12. Use the pilot image to locate the spine in three dimensions to ensure coverage of the region of interest (cervical, thoracic, lumbosacral) spine.

13. Let the patient know you are ready and begin the scan. 
Table A8.8.5 Primary Clinical Imaging Parameters for Sequence 4 ( $T_{1}$-Weighted Image)

\begin{tabular}{|c|c|}
\hline Patient position & Supine \\
\hline Scan type & Conventional spin echo \\
\hline Imaging plane (orientation) & Transverse \\
\hline Central slice or volume center & $\begin{array}{l}\text { Centered on the area of interest (as in } \\
\text { sequence } 2 \text {, Table A8.8.3) }\end{array}$ \\
\hline Echo time $\left(T_{\mathrm{E}}\right)$ & $10 \mathrm{msec}$ \\
\hline Repeat time $\left(T_{\mathrm{R}}\right)$ & $500 \mathrm{msec}$ \\
\hline Flip angle (FA) & $90^{\circ}$ \\
\hline Fields of view $\left(\mathrm{FOV}_{\mathrm{x}}, \mathrm{FOV}_{\mathrm{y}}\right)$ & As in sequence 2, Table A8.8.3 \\
\hline Resolution $(\Delta x, \Delta y)$ & $\begin{array}{l}\text { Cervical: } 0.94 \mathrm{~mm}, 0.94 \mathrm{~mm} \\
\text { Thoracic: } 1.25 \mathrm{~mm}, 1.25 \mathrm{~mm} \\
\text { Lumbosacral: } 1.09 \mathrm{~mm}, 1.09 \mathrm{~mm}\end{array}$ \\
\hline Number of data points collected $\left(N_{\mathrm{x}}, N_{\mathrm{y}}\right)$ & 256,256 \\
\hline Display matrix $\left(D_{\mathrm{x}}, D_{\mathrm{y}}\right)$ & 256,256 \\
\hline Slice thickness $(\Delta z)$ & $\begin{array}{l}\text { Cervical: } 3 \mathrm{~mm} \\
\text { Thoracic: } 3-8 \mathrm{~mm} \\
\text { Lumbar: } 4 \mathrm{~mm}\end{array}$ \\
\hline Number of slices & Varies with spinal level \\
\hline Slice gap & $\begin{array}{l}\text { Cervical: } 1 \mathrm{~mm} \\
\text { Thoracic: } 1 \mathrm{~mm} \\
\text { Lumbar: } 1 \mathrm{~mm}\end{array}$ \\
\hline Number of acquisitions $\left(N_{\text {acq }}\right)$ & 2 \\
\hline Flow compensation & Yes (if available) \\
\hline Slice locations & See text (Basic Protocol, step 16) \\
\hline Saturation pulses & No \\
\hline Scan time & $\sim 4 \min$ \\
\hline
\end{tabular}

\section{Sequence 3: Sagittal $T_{2}$-weighted fast spin echo}

14. Review the pilot scans and ensure that the saturation pulse is correctly placed anterior to above the slab of interest.

15. Run sequence 3 according to Table A8.8.4.

\section{Sequence 4: Transverse $T_{1}$-weighted conventional spin echo}

16. Using the midline sagittal $T_{1}$-weighted image acquired in sequence 2 , set the transverse acquisition parameters as follows:

a. Cervical spine: stacked images from $\mathrm{C} 1$ through C7-T1.

b. Thoracic spine: Stacked images through levels of interest

c. Lumbosacral spine: 5 slices each, angled to the plane of the intervertebral disc at L3-4, L4-5, and L5-S1; one slice each, angled to the intervertebral disc at L1-2 and L2-3.

17. Supplement additional slices according to visible disease present or to clinical query.

18. Run the sequence according to Table A8.8.5.

\section{Sequence 5: Transverse $T_{2}$-weighted fast spin echo}

Post Surgical Spinal Evaluation

A8.8.6

19. Using the midline $T_{1}$-weighted image acquired in sequence 2 , repeat the setup as in Table A8.8.6.

20. Run sequences according to Table A8.8.6. 
Table A8.8.6 Primary Clinical Imaging Parameters for Sequence $5\left(T_{2}\right.$-Weighted Image, FSE) ${ }^{a}$

\begin{tabular}{ll}
\hline Patient position & Supine \\
Scan type & Fast spin echo \\
Imaging plane (orientation) & Transverse \\
Central slice or volume center & Centered on the region of interest (as \\
& in sequence 2, Table A8.8.3) \\
Echo time $\left(T_{\mathrm{E}}\right)$ & $100 \mathrm{msec}$ \\
Echo train length $(\mathrm{ETL})$ & 8 \\
Repeat time $\left(T_{\mathrm{R}}\right)$ & $4000 \mathrm{msec}$ \\
Flip angle $(\mathrm{FA})$ & $90^{\circ}$ \\
Fields of view $\left(\mathrm{FOV}, \mathrm{F}_{\mathrm{x}}, \mathrm{FOV}_{\mathrm{y}}\right)$ & As in sequence 2, Table A8.8.3 \\
Resolution $(\Delta x, \Delta y)$ & Cervical: $0.94 \mathrm{~mm}, 0.94 \mathrm{~mm}$ \\
& Thoracic: $1.25 \mathrm{~mm}, 1.25 \mathrm{~mm}$ \\
& Lumbosacral: $1.09 \mathrm{~mm}, 1.09 \mathrm{~mm}$ \\
Number of data points collected $\left(N_{\mathrm{x}}, N_{\mathrm{y}}\right)$ & 256,256 \\
Display matrix $\left(D_{\mathrm{x}}, D_{\mathrm{y}}\right)$ & 256,256 \\
Slice thickness $(\Delta z)$ & Cervical: $3 \mathrm{~mm}$ \\
& Thoracic: $3-8 \mathrm{~mm}$ \\
Number of slices & Lumbar: $4 \mathrm{~mm}$ \\
Slice gap & Varies with spinal level \\
& Cervical: $1 \mathrm{~mm}$ \\
Number of acquisitions $\left(N_{\mathrm{acq}}\right)$ & Thoracic: $1-2 \mathrm{~mm}$ \\
Slice locations & Lumbar: $1 \mathrm{~mm}$ \\
Flow compensation & 2 \\
Saturation pulses & See text $($ Basic Protocol, step 16) \\
Scan time & Yes (if available) \\
\hline
\end{tabular}

${ }^{a}$ FSE: fast spin echo.

Sequence 6 and 7: Sagittal (sequence 6) and transverse (sequence 7) i.v. contrast enhanced $T_{1}$-weighted spin echo

21. Remove patient from the magnet.

22. Establish an i.v. line from which the contrast agent can be injected, and attach this line securely to the patient so that movement into or out of the magnet will not pull at the patient's arm. Push the patient back to the magnet.

It is preferable to insert the line prior to imaging and to leave the patient in the magnet, with no intervening motion, between the scans run before contrast agent injection and those run after injection.

\section{Scan pilot}

23. Run a rapid three-plane positioning pilot scan (see sequence 1).

\section{Scan sequences}

24. Leaving the patient in the magnet, inject the contrast agent, flush the i.v. line with 10 cc saline, and then immediately run sagittal (sequence 6) and transverse (sequence 7) $T_{1}$-weighted image sequences (see sequences 2 and 4; sequences 6 and 7 are the same as sequences 2 and 4, respectively).

A dose of $0.1 \mathrm{mmol} / \mathrm{kg}$ of contrast agent is usually given i.v.

Fat-suppression techniques may be very useful in order to suppress osseous vertebral marrow fat and perispinal soft tissue fat, thereby clearly distinguishing enhancement from normal fatty tissue. 


\section{CORONAL CONTRAST ENHANCED ACQUISITION}

In some instances, a coronal acquisition may be helpful to analyze the perispinal tissues for infectious phlegmon or frank abscess formation.

\section{Sequence 8: Contrast enhanced coronal $T_{1}$-weighted image}

Run the sequence according to sequence 8, Table A8.8.7. Fat suppression may be useful.

Table A8.8.7 Primary Clinical Imaging Parameters for Sequence 8 ( $T_{1}$-Weighted Image)

\begin{tabular}{ll}
\hline Patient position & Supine \\
Scan type & Conventional spin echo \\
Imaging plane (orientation) & Coronal \\
Central slice or volume center & Centered on the region of interest (as \\
& in sequence 2, Table A8.8.3) \\
Echo time $\left(T_{\mathrm{E}}\right)$ & $10 \mathrm{msec}$ \\
Repeat time $\left(T_{\mathrm{R}}\right)$ & $500 \mathrm{msec}$ \\
Flip angle $(\mathrm{FA})$ & $90^{\circ}$ \\
Fields of view $\left(\mathrm{FOV}_{\mathrm{x}}, \mathrm{FOV}_{\mathrm{y}}\right)$ & As in sequence 2, Table A8.8.3 \\
Resolution $(\Delta x, \Delta y)$ & Cervical: $0.94 \mathrm{~mm}, 0.94 \mathrm{~mm}$ \\
& Thoracic: $1.25 \mathrm{~mm}, 1.25 \mathrm{~mm}$ \\
& Lumbosacral: $1.09 \mathrm{~mm}, 1.09 \mathrm{~mm}$ \\
Number of data points collected $\left(N_{\mathrm{x}}, N_{\mathrm{y}}\right)$ & 256,256 \\
Display matrix $\left(D_{\mathrm{x}}, D_{\mathrm{y}}\right)$ & 256,256 \\
Slice thickness $(\Delta z)$ & $3-4 \mathrm{~mm}$ \\
Number of slices & Varies with spinal level \\
Slice gap & $1-2 \mathrm{~mm}$ \\
Number of acquisitions $\left(N_{\mathrm{acq}}\right)$ & 2 \\
Slice location & From front to back of spinal column \\
Saturation pulses & No \\
Fat suppression & Yes: fat saturation or STIR (short tall \\
& inversion recovery) \\
Scan time & $\sim 4$ min \\
\hline
\end{tabular}

\section{COMMENTARY}

\section{Background Information}

Despite the relatively loose application of criteria for judging operative success, lumbosacral spinal surgery has been so often unsuccessful (10\% to $40 \%)$ that failed back surgery is now labeled as a syndrome- - the failed back surgery syndrome (FBSS). In general, surgery for lumbar disc herniation relieves pain in most patients, and produces good long-time outcome in almost $90 \%$ of patients. Repeat surgery, however, is less successful, and according to several studies only $60 \%$ to $82 \%$ of patients with recurrent disc herniation improve after surgery. In patients who only have epidural scar tissue, the success rate of reintervention is as low as $17 \%$ to $38 \%$. Therefore, the obvious solution to this problem is to attempt to avoid the first operation that statistically is likely to lead to a less than satisfactory result, and thus not create a clinical condition that requires repeat spinal surgery.

The FBSS is characterized by postsurgical intractable pain in the low back and lower extremity or extremities, combined with varying degrees of functional incapacitation. The major identifiable causes of the FBSS include "clinically relevant" epidural fibrosis (i.e., fibrosis causing perineural constriction, tethering of the nerve root), recurrent or residual disc herniation, postoperative spinal infection, sterile arachnoiditis (Fig. A8.8.1), postsurgical pseudomeningocele formation and lateral recess, and foraminal or central spinal stenosis that may preexist or follow the spinal surgery. Other less common causes of the FBSS include postoperative hemorrhage, sur- 
A

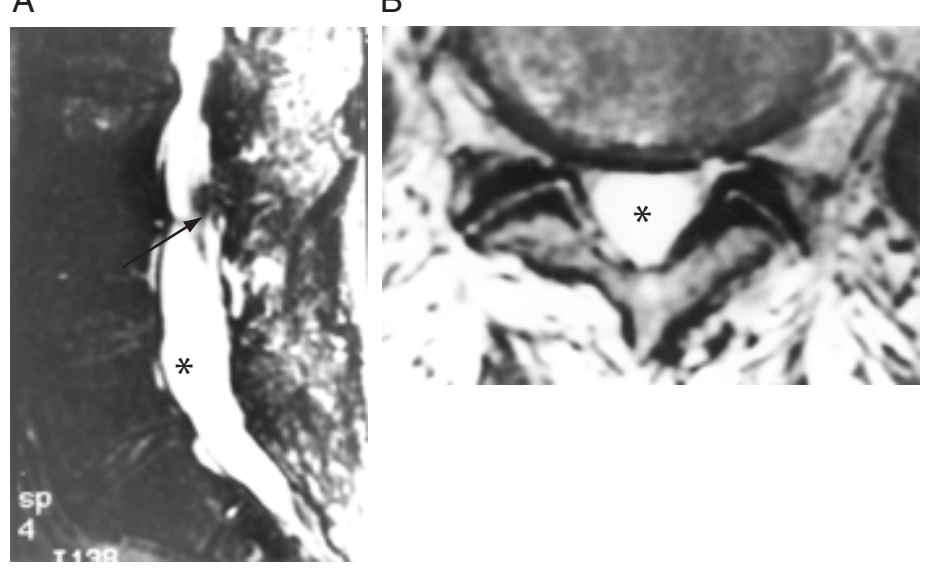

Figure A8.8.1 Postoperative chronic adhesive arachnoiditis. (A) Sagittal $T_{2}$-weighted $\left(T_{\mathrm{R}}=4000\right.$ msec, $T_{\mathrm{E}}=100 \mathrm{msec}$ ) fat suppressed image shows matting of the nerve roots superiorly (arrows) and are "empty thecal sac" inferiorly (asterisk) indicating adhesive fibrosis of the contents of the thecal sac. (B) Transverse $T_{2}$-weighted $\left(T_{R}=4000 \mathrm{msec}, T_{E}=100 \mathrm{msec}\right.$ ) image at $L 5$ shows again the "empty thecal sac" (asterisk) in this region due to adhesion of the intrathecal nerve roots to the peripheral walls of the thecal sac.

gery inadvertently performed on the wrong side or at the incorrect segmental level, direct nerve injury at the time of surgery, spinal pain of mechanical origin, e.g., posterior spinal facet (i.e., zygapophyseal) joint disease, and attempted surgical fusion failure. Still further causes of the FBSS are recurrent or residual clinical symptoms related to anterior spinal disc protrusion, spinal nerve root sterile radiculitis (i.e., neuritis), disc herniation at a spinal level other than that operated on, posterior facet joint fracture, and progressive spinal instability with attendant spondylolisthesis. These conditions should be distinguished from acceptable and expected postoperative findings found after successful lumbar surgery (i.e., that surgery associated with clinical sign and symptom relief).

\section{Critical Parameters and Troubleshooting}

Cerebrospinal fluid (CSF) flow, cardiac, laryngeal, body wall, and other sources of motion can produce artifacts that can on occasion significantly degrade the images. Proper spatial (e.g., prevertebral) saturation pulses and sometimes flow compensation pulses and/or cardiac/respiratory gating can reduce these artifacts significantly. In many instances these artifacts may be difficult or impossible to easily overcome from patient to patient.

The initiation of MR imaging within $\sim 2 \mathrm{~min}$ of the i.v. gadolinium injection is important because some disc herniations can be somewhat vascularized and therefore may enhance relatively early. The practical basis for this imaging strategy is that the vessels in the scar tissue are relatively homogeneously distributed, whereas in disc herniation the vessels are quite heterogeneous or are centrally absent. Therefore a centrally nonenhancing epidural mass would be labeled a partially vascularized disc herniation (Fig. A8.8.2), but homogeneous enhancement of an epidural process would be termed epidural fibrosis. It should be noted that imaging after 20 to $30 \mathrm{~min}$ of the i.v. contrast agent administration is not helpful because many recurrent disc herniations enhance more or less homogeneously within this delayed time frame as the gadolinium progressively seeps into the extruded disc material.

Intravenously administered gadolinium compounds are also an important adjunct to the MR evaluation of the postoperative lumbosacral spine in the elucidation and differentiation of spinal, leptomeningeal, and/or neural inflammation (infectious or aseptic). The proper differentiation of these varied pathologic phenomena on MR imaging should allow improved patient triage toward appropriate medical-surgical therapy aimed at treating the specific pathologic change.

\section{Anticipated Results}

It should be clear to those who perform and interpret medical images of the spine following
Extradural Spine

A8.8.9

Supplement 8 
A

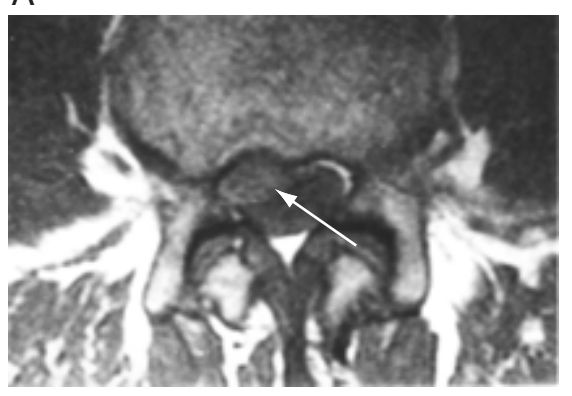

B

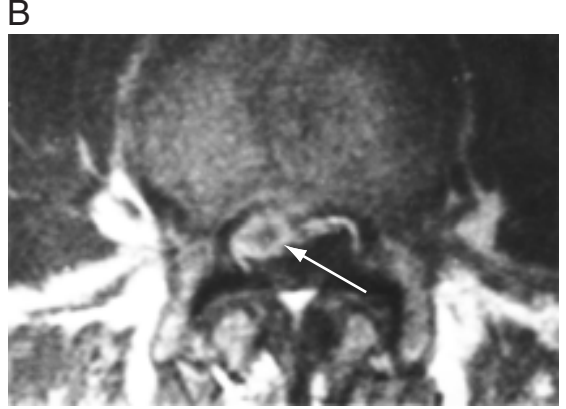

Figure A8.8.2 Postoperative recurrent disc herniation. (A) Transverse $T_{1}$-weighted ( $T_{\mathrm{R}}=500$ msec, $T_{E}=10 \mathrm{msec}$ ) image shows mildly hyperintense mass (arrow) at L4-5 on the right side of the spinal canal anteriorly. (B) i.v. gadolinium enhanced $T_{1}$-weighted $\left(T_{\mathrm{R}}=500 \mathrm{msec}, T_{E}=10 \mathrm{msec}\right)$ image shows rim enhancement surrounding a recurrent disc herniation (arrow).

one or more forms of surgical therapy that the images are often difficult to interpret, in part because of the superimposition of the original disease process, alteration engendered by the surgery and/or because of a complication of the surgical procedure. Although long-term experience in this area is without a doubt helpful in regard to improving interpretive skills, nevertheless, certain sequellae can be predicted regardless of the interpreter's background.

Once the normal or expected post-surgical findings are understood, the subtle and gross changes that depart from these observations can be better analyzed. The importance of a high level of competence in the domain of posttherapeutic neurodiagnostic imaging is in the knowledge that the patient returning for restudy may be acutely in distress or even in medical danger (e.g., postoperative spondylitis). In fact, the clinical presentation post-therapeutically may well be more severe or dire than was observed pretherapeutically. Thus, an in-depth appreciation of the broad range of clinicoradiologic possibilities, as presented here, should place the medical imaging physician in an excellent position to provide an experienced diagnostic evaluation in the patient presenting with recurrent or new signs and symptoms following any one of the spectrum of possible spinal surgical procedures.

\section{Literature Cited}

Ghazi, J., Golimbu, C.N., and Engler, G.L. 1992. MRI of spinal fusion pseudoarthrosis. J. Comput. Assist. Tomogr. 16:324-326.

Grane, P., Josephsson, A., Seferlis, A., and Tullberg, T. 1998. Septic and aseptic post-operative discitis in the lumbar spine-evaluation by MR imaging. Acta Radiol. 39:108-115.

Jinkins, J.R. 1993. Magnetic resonance imaging of benign nerve root enhancement in the unoperated and postoperative lumbosacral spine. Neuroimaging Clin. North Am. 3:525-541.

Jinkins, J.R., Osborn, A.G., Garrett, D., Jr., and Hunt, S. 1993. Spinal nerve enhancement with Gd-DTPA: MR correlation with the postoperative lumbosacral spine. Am. J. Neuroradiol. 14:383-394.

Shafaiae, F., Bunscuh, C., and Jinkins, J.R. 1997. The posttherapeutic lumbosacral spine. In Posttherapeutic Neurodiagnostic Imaging (J.R. Jinkins, ed.) pp. 223-243. Lippincott-Raven. Philadelphia.

Shellock, F.G. 1996. Pocket Guide to MR Procedures and Metallic Objects. Lippincott-Raven, Philadelphia.

Van Goethem, J.W., Parizel, P.M., van den Hauwe, L., and DeSchepper, A.M. 1997. Imaging findings in patients with failed back surgery syndrome. J. Belge Radiol. 80(2):81-84.

Contributed by J. Randy Jinkins and David D. Stark

Downstate Medical Center

State University of New York

Brooklyn, New York
Post Surgical Spinal Evaluation 